\title{
Assessing the Feasibility of Using a Multi-Modal Simulation Approach to Prepare Nurse Practitioners in Primary Health Care
}

\author{
Eric Staples' ${ }^{1}$ Joanna Pierazzo ${ }^{2}$ \\ ${ }^{1}$ RN, DNP, Independent Nursing Practice Consultant, Hamilton, ON, Canada \\ ${ }^{2}$ RN, PhD, Assistant Professor, McMaster University, Hamilton, ON, Canada \\ Email: eric.staples@sympatico.ca, pierazj@mcmaster.ca
}

Received 31 May 2016; accepted 19 July 2016; published 22 July 2016

Copyright (C) 2016 by authors and OALib.

This work is licensed under the Creative Commons Attribution International License (CC BY). http://creativecommons.org/licenses/by/4.0/

(c) (i) Open Access

\begin{abstract}
Simulation based learning in nursing education provides learners with opportunities to practice real-life experiences. Enhancing the education of nurse practitioners (NPs) with simulation based teaching and learning strategies has not been well investigated. There is limited evidence related to learning outcomes and the use of high fidelity simulation or standardized patients. In an Ontario Primary Health Care Nurse Practitioner (PHCNP) Program, the use of a multi-model simulation learning activity was piloted with a group of NP learners. The learning activity consisted of three scenarios, each representing typical conditions seen in primary health care across the lifespan. Each scenario was carefully developed with consideration of curriculum goals, use of simulation technology or standardized patients, and the role of faculty facilitators. Learners worked in pairs as a team to complete a focused history and physical examination, formulate a diagnosis, and develop a plan of care or action for the patients. Following each of the three scenarios, the learner teams received focused feedback on their performance. A guided group reflection was conducted following the learning activity. The feedback from the learners was positive, with a recommendation to include similar learning opportunities earlier in the NP curriculum. The learners valued the active learning process, including peer collaboration and group debriefing. Although the findings from this pilot included a small group of learners, there are valuable considerations for nursing faculty teaching in NP programs with a primary health care focus.
\end{abstract}

\section{Keywords}

Simulation Based Learning, Curriculum Evaluation, Advanced Practice Nursing, Nurse Practitioner, Primary Health Care

Subject Areas: Nursing

How to cite this paper: Staples, E. and Pierazzo, J. (2016) Assessing the Feasibility of Using a Multi-Modal Simulation Approach to Prepare Nurse Practitioners in Primary Health Care. Open Access Library Journal, 3: e2779.

http://dx.doi.org/10.4236/oalib.1102779 


\section{Introduction}

Since the inception of the Primary Health Care Nurse Practitioner (PHCNP) consortium in Ontario in 1995, learning activities within the formal curriculum have primarily utilized low-fidelity simulation in the form of, faculty demonstration and student return demonstration for skill acquisition, standardized patients for teaching the Papanicolaou (Pap) screening procedure, and performance examinations, such as objective structured clinical examinations (OSCEs). Some consortium sites have formalized internal, standardized patient (SP) programs, while others have elected to hire patient actors. Several barriers to advancing technology use, specifically high-fidelity simulation have included limited access to technologists; limited faculty resources and expertise; competition for access to equipment and laboratory space; perceived lack of relevancy to primary health care; and fiscal constraints for purchasing high-fidelity simulation equipment and developing curricular content. The purpose of this educational initiative was to explore the feasibility of enhancing PHCNP education at one site in the 9-university consortium, using a multi-modal simulation approach with a focus on learner engagement and guided group learning.

\section{High-Fidelity Simulation and Primary Health Care Nurse Practitioner Education}

In nursing education, clinical learning activities provide real experiences and opportunities for the transfer of learning to common practice situations [1]. Over the last decade, with the proliferation of simulation technology, educators are better able to develop learning activities to complement low frequency and higher acuity clinical situations. Simulation is an educational technique in which elements of the real world are appropriately integrated to achieve specific goals related to learning or assessment [2], including clinical reasoning and decision-making [3]. This teaching and learning strategy may include the use of multiple simulation modalities such as SPs who are trained actors, computer-based virtual programs, simulation board games, partial task trainers and human-like mannequins.

High-fidelity simulation learning activities commonly utilize computer-operated human-Like mannequins with physiologic and vocal responses that can be programmed to respond to the learner's actions [4]. Several advantages have been identified to using high-fidelity simulation, including the ability to facilitate clinical scenario conditions and events that cannot be replicated in the clinical practice setting; providing a safe learning environment for the learner; providing a consistent experience for all learners; and facilitating scheduled integration within a curriculum [5] [6].

Simulation, as a teaching and learning strategy, has become widely accepted across the spectrum of health care education and practice institutions in disciplines such as nursing, midwifery, physical therapy, pharmacy, and medicine [5]-[8]. Although the literature describes the use of high-fidelity simulation in NP programs, it is most often associated with acute care nurse practitioner (ACNP) programs for technical skills such as ACLS, intubation, invasive line insertion, and for treatment of hypovolemic and septic shock, and malignant hyperthermia [9]. In some instances, it has been suggested the use of simulation learning may substitute for a loss of preceptored clinical experiences [10].

At present, evidence related to the use of high-fidelity simulation in formal PHCNP programs is limited, with a primary focus on the provision of patient care within a primary health care framework including skills related to communication, physical assessment, health education, clinical decision-making, and the management and investigation of health issues. Within this primary health care context, NPs require knowledge and expertise to manage potential exacerbation of patient symptoms. In some situations, patients may become more acute and require NP interventions to facilitate access to acute care facilities. With the integration of experiential learning experiences such as high fidelity simulation, PHCNP learners may further enhance their diagnostic reasoning competencies, engage in self-reflection, and identify knowledge gaps for further learning [11].

\section{Simulation Learning Context}

The site for this educational initiative took place at the Centre for Simulation Based Learning (CSBL) in the Faculty of Health Sciences at McMaster University, Hamilton, Ontario. This interprofessional education centre provides support in assisting health profession programs to deliver the full spectrum of simulation modalities. In developing this initiative, faculty with expertise in the PHCNP curriculum and simulation-based learning collaborated with the CSBL to plan the learning activities. This included scenario development, training of stan- 
dardized patients and organization of the setting, such as technology, room set-up, props and materials. Three simulation rooms were used; each arranged to resemble a typical primary health care examination room. In order to provide an opportunity for student assessment, each room had a two-way mirror for viewing. Behind the mirror, each room housed what was required to enact each patient scenario, including computer, video, and sound equipment for the patient simulator.

\section{The Simulation Scenarios}

Three scenarios (one per simulation room) were developed for the simulation learning experience; each illustrated a typical condition seen in primary health care across the lifespan. With the technical support of the CSBL, the scenarios were programmed to direct specific clinical responses and, to respond to various interventions by the learner. The goal of each simulation station was to provide students with an opportunity to engage in learning about specific competencies within the PHCNP curriculum including, therapeutic communication, assessment, formulation of a differential diagnoses, and the development of a management plan.

The first scenario described an 84-year-old female, eight weeks post total hip replacement, living in a nearby retirement home who was complaining of chest discomfort, non-productive cough, and an upset stomach over the past two days. The learning goal of this scenario was to recognize an emergent, evolving cardiac event and the need for transfer to the hospital. The second scenario illustrated an18 month-old male infant, accompanied by his mother with a two-day history of vomiting, diarrhea, fever, and lethargy. The learning goal of this scenario was to assess the infant, but also to complete a brief family assessment. The third scenario utilized only a standardized patient and described a retired widow who was about to embark on an extended holiday. She had been seen five weeks earlier for a lump in her right groin, and had returned for follow-up after a computerized tomography (CT) scan of the abdomen, which confirmed advanced metastatic colon cancer. The learning goal of this scenario was to communicate the diagnosis, encourage deferral of the planned trip, and plan for an oncology referral.

\section{Simulated Learning Encounter}

There were six learners who volunteered to participate in this pilot, all whom were in their final clinical practicum course in the PHCNP program. The experience was organized similar to an Objective Structured Clinical Examination (OSCE), with the three stations each representing one of the patient scenarios. The learners rotated through each station in pre-assigned pairs. All learners signed, per university policy, a confidentiality agreement and a photo/video/film release form. The simulations were videotaped which were later used as part of the debriefing process and for evaluating the overall learning experience.

The learners readily volunteered to participate in the pilot. They began by reviewing the learning goals, and relevant readings and course materials. Since the students had previously not been exposed to a high-fidelity simulation experience, a 10-minute faculty-led orientation was provided about the use of the simulator. This included an explanation of the equipment and room set-up, the flow of the learning activity including the timeframe for each scenario, and finally, the debriefing and evaluation process at the conclusion of the activity. The learners were aware there would be faculty facilitating each of the stations as well as the debriefing process. Importantly, it was explained the learning activity would not be graded, although would offer an opportunity to enhance clinical learning and assist faculty to understand the use of a multi-modal simulation approach within a PHCNP curriculum.

During the simulations, learner pairs were expected to work as a team to gather data through a focused history and physical examination, formulate a diagnosis, and develop a plan of care or action for the "patient". They were given a total of 30minutes for each scenario, which included 10 minutes for orientation to the simulation mannequin, about 15 minutes to run the scenario for each pair, and 5 minutes to debrief about debriefing about their perceptions of the case scenario.

Similar to an OSCEs, a brief description of the patient was provided in writing to each learner pair prior to entering the room. The description provided enough detail to instruct the learner through the patient encounter. In addition, information related to medications, and relevant laboratory results were made available for the learners to review. When the learner pair felt ready to begin, they initiated the patient encounter interview. During the simulation, the faculty were situated behind the two-way mirror. As required, the faculty participated as either the patient's voice or as the controller, manipulating the simulator responses. The faculty also assessed learner performance, noting relevant video footage for the debriefing experience. 
An important consideration in establishing a level of realism for the learners was to ensure the appropriate simulation modality was selected for each of the scenarios. In the older adult scenario, the adult simulator was placed in a wheelchair beside an examination table, and in the infant scenario, the infant simulator was placed on the examination table, and a standardized patient, acting as the mother, was also in the room. This third scenario consisted of a standardized patient in a room with an examination table.

\section{Group Debriefing}

At the end of the simulation experience, the faculty met with the six learners to facilitate are flective discussion about the learning experience. The goal was to receive learner feedback and reflection on the value of the experience. Questions included: How did the scenario make you feel as an NP learner? What did you feel you did well? Where did you feel you needed to improve? As learners shared their perceptions and feelings, the faculty facilitated learning related to scenario content, and also guided a process to understand the learners' learning experience. The faculty provided constructive feedback on what they assessed, including therapeutic communication with the simulator or in the case of the infant, with the mother, as well as their plan of care for each patient i.e., admission to ER or pharma therapeutic management. Within the debriefing process, the faculty also shared relevant video footage to enhance learner reflection and promote discussion.

\section{Learner Feedback and Analysis of Responses}

All learners, by policy, who engage in a simulation activity, were asked to complete a Simulation Feedback Form (see Figure 1). The tool consists of five statements ranked on a Likert scale: 1 = very poor; 2 = poor; 3 = average; 4 = good; 5 = very good with additional space for comments. The final statement provided space for learner comments related to their learning experience.

Overall, the feedback received from learners related to the simulation experience was positive with no one rating the experience as very poor or poor. The learners were divided on whether the physical set-up looked realistic, scoring equally between 3 to 5 . Half of the group rated the equipment average for running smoothly. During the adult simulation, there were audio difficulties, and anatomical locations of pulses on the simulator were different from a normal human. This made pulses and heart sounds difficult for learners to landmark and evaluate.

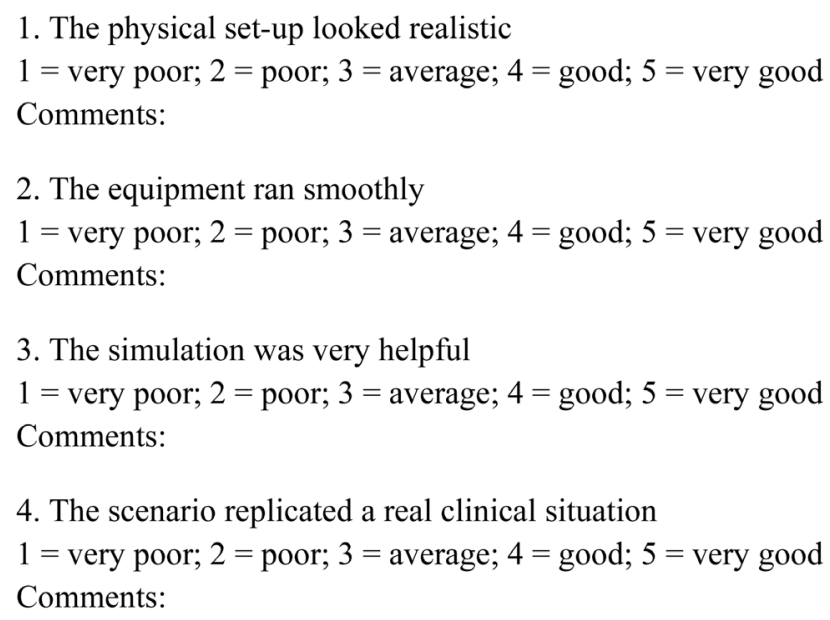

5. My overall experience was:

(Adapted from McMaster University Centre for Simulation-Based Learning Feedback

Form, 2012)

Figure 1. Simulation feedback form. 
$83 \%$ of the learners rated the experience very good as being very helpful; $67 \%$ rated the scenarios as very good in replicating real clinical situations they would see in primary health care practice, and $67 \%$ of learners rated the overall experience as good. One learner commented that it was a "great learning experience!".

In the large group debriefing, the comments shared by the learners were summarized. Learners commented favorably for the opportunity to have a high-fidelity simulation stating they would have preferred this learning strategy earlier in the course. There were comments requesting more simulation time in the program, as well as one-on-one skill practice time with a variety of patient scenarios. Several learners commented simulation activities should be added to the advanced health assessment course. Only one learner reported the simulation was not very helpful and another stated that she had difficulty thinking during the simulation. In summary, the majority felt the use of simulation should continue and be adjunctive in the curriculum. The ability to collaborate with peers during the simulation, engage in a debrief after the experience, and be able to ask questions was considered very positive.

\section{Discussion}

A primary goal in nursing education is to assess, develop, implement, and evaluate teaching/learning methodologies that better prepare learners for advanced clinical nursing practice. This learning initiative demonstrated the possible potential of incorporating primary health care patient scenarios with a multi-modal simulation approach ranging from high-fidelity simulation to a hybrid model of high-fidelity simulation with a standardized patient. The hybrid simulation model would provide learners with exposure to patient scenarios that are more representative of the primary health care context. In addition, it does allow for varied learning modalities that may be beneficial in enhancing learner confidence, self-efficacy, and diagnostic reasoning skills.

The integration of simulation in a PHCNP curriculum is a relevant consideration for NP educators. Although the learners who participated in this initiative identified some positive strengths, it would be important to consider the cost implications in developing scenarios, training of standardized patients, and logistics for all learners to participate. In addition, adding high-fidelity simulation required time for faculty to collaborate with the simulation centre staff, so that the scenarios were appropriately sequenced and the room set-up was realistic.

At the conclusion of the learning experience, faculty reflected on the overall experience and learner feedback to ascertain offering the experience again. Based on this reflection, it was determined that increased time for the simulation experience was needed, so that learners and faculty do not feel rushed during the learning process. It was also recognized that a more structured approach to the debriefing session may beneficial. This would ensure all learners receive the same information, the objectives of the simulation have been met, and there is enough time for an effective, guided reflection.

Faculty did not have a pre- and post-test measure of the learner's learning during the simulation experience to compare what the learner knew before and after the simulation. In the future, it may be helpful to assess learner's knowledge before and after the simulation experience.

This will allow faculty to evaluate whether learners' knowledge improved after the experience and whether there is a need to adjust the patient scenarios to improve learning. In addition to knowledge, it may have been beneficial to further assess learner characteristics, such confidence or self-efficacy. The evaluation tool used to attain feedback was only used to assess learners' perceptions about the experience and their overall learning. In the future, one consideration is to incorporate a learning outcomes' assessment tool. One example is the Simulation Effectiveness Tool (SET) [12], consisting of 13 statements scored on a three-point Likert-type scale ranging from do not agree to strongly agree. SET is designed to measure three aspects of learning outcomes: skills or knowledge gained because of the simulated cases, confidence level and satisfaction attitudes.

\section{Conclusion}

The simulation experience allowed PHCNP learners the opportunity to apply theory learned in the classroom with competencies attained in the clinical practice setting in a controlled simulated learning environment, while receiving faculty feedback. Moreover, using a multi-modal simulation approach provided learners the opportunity to reflect on clinical areas where they may have had limited exposure. The faculty involved in the simulation expressed high satisfaction with the learning process and felt it provided an opportunity to augment the teacher-learner interaction. Faculty teaching in NP programs with a primary health care focus may benefit from information about high-fidelity simulation to assist them in preparing future competent NPs. 


\section{References}

[1] Oermann, M.H. and Gaberson, K.B. (2009) Evaluation and Testing in Nursing Education. Springer, New York.

[2] Gaba, D.M. (2004) The Future Vision of Simulation in Health Care. Quality \& Safety in Health Care, 13, i2-i10. http://dx.doi.org/10.1136/qshc.2004.009878

[3] Seropian, M., Brown, K., Gavilanes, J. and Driggers, B. (2004) An Approach to Simulation Program Development. Journal of Nursing Education, 43, 170-174.

[4] Nehring, W. and Lashley, F. (2007) High-Fidelity Patient Simulation in Nursing Education. Bartlett \& Jones, Sudbury.

[5] Branch, C. (2013) Pharmacy Students' Learning and Satisfaction with High-Fidelity Simulation to Teach DrugInduced Dyspepsia. American Journal of Pharmaceutical Education, 77, 1-9. http://dx.doi.org/10.5688/ajpe77230

[6] Weller, J.M., Bloch, M., Young, S., Maze, M., Oyesola, S., Wyner, J., et al. (2003) Evaluation of High Fidelity Patient Simulator in Assessment of Performance of Anesthetists. British Journal of Anesthesia, 90, 43-47. http://dx.doi.org/10.1093/bja/aeg002

[7] Shoemaker, M.J., Riemersma, L. and Perkins, R. (2009) Use of High Fidelity Human Simulation to Teach Physical Therapist Decision-Making Skills for the Intensive Care Setting. Cardiopulmonary Physical Therapy Journal, 20, 13-18.

[8] Cooper, J.B., Singer, S.J., Hayes, J., Sales, M., Vogt, J.W., Raemer, D. and Meyer, G.S. (2011) Design and Evaluation of Simulation Scenarios for A Program Introducing Patient Safety, Teamwork, Safety Leadership, and Simulation to Healthcare Leaders and Managers. Society for Simulation in Healthcare, 6, 231-238. http://dx.doi.org/10.1097/SIH.0b013e31821da9ec

[9] Pittman, O.A. (2012) The Use of Simulation with Advanced Practice Nursing Students. American Academy of Nurse Practitioners, 24, 516-520. http://dx.doi.org/10.1111/j.1745-7599.2012.00760.x

[10] Mompoint-Williams, D., Brooks, A., Lee, L., Watts, P. and Moss, J. (2014) Using High-Fidelity Simulation to Prepare Advanced Practice Nursing Students. Clinical Simulation in Nursing, 10, e5-e10. http://dx.doi.org/10.1016/j.ecns.2013.07.005

[11] Clapper, T. (2010) Beyond Knowles: What Those Doing Simulation Need to Know about Adult Learning Theory. Clinical Simulation in Nursing, 6, 37-e14. http://dx.doi.org/10.1016/j.ecns.2009.07.003

[12] Elfrink Cordi, V.L., Leighton, K., Ryan-Wenger, N., Doyle, T.J. and Ravert, P. (2012) History and Development of the Simulation Effectiveness Tool (SET). Clinical Simulation in Nursing, 8, e199-e210.

http://dx.doi.org/10.1016/j.ecns.2011.12.001

\section{Submit or recommend next manuscript to OALib Journal and we will provide best service for you:}

- Publication frequency: Monthly

- 9 subject areas of science, technology and medicine

- Fair and rigorous peer-review system

- Fast publication process

- Article promotion in various social networking sites (LinkedIn, Facebook, Twitter, etc.)

- Maximum dissemination of your research work

Submit Your Paper Online: Click Here to Submit

Contact Us: service@oalib.com 